hep-th/9511126

ITEP-M $/ 95$

OU-HET-227

\title{
INTEGRABILITY and SEIBERG-WITTEN THEORY Curves and Periods
}

\author{
H.Itoyama ${ }^{1}$ and A.Morozov ${ }^{2}$
}

\begin{abstract}
Interpretation of exact results on the low-energy limit of $4 d N=2 \mathrm{SUSY}$ YM in the language of $1 d$ integrability theory is reviewed. The case of elliptic Calogero system, associated with the flow between $N=4$ and $N=2$ SUSY in $4 d$, is considered in some detail.
\end{abstract}

\footnotetext{
${ }^{1}$ Departement of Physics, Graduate School of Science, Osaka University, Toyonaka, Osaka 560, Japan. E-mail address: itoyama@funpth.phys.sci.osaka-u.ac.jp

${ }^{2} 117259$, ITEP, Moscow, Russia. E-mail address: morozov@vxitep.itep.ru
} 


\section{Contents}

1 Generalities 2

$2 \quad 4 d$ Physics $\quad 3$

3 Integrability theory 4

4 Examples 6

4.1 Free particles (classical module space) $\ldots \ldots \ldots \ldots \ldots$

4.2 Toda-chain system (quantum module space in the absence of matter) $\ldots \ldots \ldots$

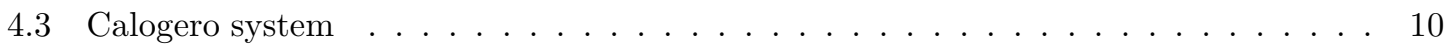

4.3.1 Elliptic Calogero (quantum moduli space in the UV-finite case) . . . . . . . . 10

4.3.2 Appendix. Comparison with Donagi-Witten polinomials . . . . . . . . . . 16

4.3.3 Appendix. Rational Calogero (cusp singularity on the bare spectral curve) . 18

4.4 From elliptic Calogero (gauge $N=4$ ) to Toda-chain (gauge $N=2$ without matter) 19

5 Conclusion 21

6 Acknowledgements 21 


\section{Generalities}

One of the newly emerging paradigmas of modern quantum theory is identification of exact effective actions (EA) and (generalized) integrable systems [1]. ${ }^{1}$ The motivation for this comes from the large reparametrization symmetry peculiar to EA, and is rather general. However, until recently too few examples were known of exactly evaluated EA, and most of evidence to confirm this view was emerging from the study of matrix models, which are at best $1+1$-dimensional quantum theories. Recently, in a brilliant breakthrough, N.Seiberg and E.Witten $[2,3]$ evaluated exact low-energy effective actions in certain ( $N=2$ SUSY YM + matter) $3+1$ dimensional theories. With no surprise, it was demonstrated in [4] that the answers from $[2,3]$ possess natural interpretation in terms of integrable systems.

What is impressive, in this particular context just the old-known types of integrable systems, associated with finite-dimensional Lie algebras (rather than with affine and multi-loop ones) appeared relevant. It was suggested in [4] that the reason for this can be the pecularity of low-energy effective actions, which are obtained from generic EA by specific Bogolubov-Whitham averaging procedure, invloving a limit $\frac{\text { normalization point }}{\text { all non-vanishing masses }} \longrightarrow 0$. As usual, after a limit is taken, different original models can get into the same universality class and, presumably, a large variety of such classes is represented by Whitham theories on spectral surfaces of complex dimension one.

It deserves mentioning that so far the theory of effective actions, as well as of their low-energy limits, was never addressed in a general context,- apart from the study of particular models. Therefore one should hardly be surprised that the usual intuition does not help here, and some strangelylooking phenomena occur (like emergency of Riemann surfaces in the study of dynamics of $4 d$ theories). This subject deserves more attention and hopefully dynamical mechanisms responsible for the Seiberg-Witten prescriptions will once be brought to light in these investigations.

The purpose of this paper is to review the existing evidence for identification of the SeibergWitten description with the data from integrability theory on the lines of [4] and numerous subsequent papers $[5,6,7,8,9,10,11]$.

\footnotetext{
${ }^{1}$ (Wilsonian) effective action is a functional of background fields and coupling constants. From the point of view of integrable systems (defined in terms of representation cathegories of Lie groups) these are identified as "moduli" and "time"-variables respectively, while EA is appropriate $\tau$-function. It is classical integrability that plays role here. "Quantum integrability" in such context is considered as an example of the "classical" one, associated with quantum groups. It is actually be important for description of generic (not just low-energy) effective actions, when background fields (moduli) - and thus EA themselves - are operator-valued and subjected for further averaging over "slow" quantum fluctuations.
} 


\section{$2 \quad 4 d$ Physics}

According to $[2,3]$ the low-energy description of any model of $4 d N=2$ SUSY $Y M+$ matter theory with the gauge group $G$ is encoded in the following data:

1) Riemann surface (complex curve) $\mathcal{C}_{G}\left(h_{k} \mid \tau\right)$;

2) Integrals $S_{C}=\oint_{C} d S$ along non-contractable contours (1-cycles) on this surface.

This data is enough to reconstruct exact low-energy effective action. The whole pattern depends on the moduli, $h_{k}, k=1 \ldots r_{G}$ (if there are no matter supermultiplets, otherwise their masses should also be included), which are interpreted as vacuum expectation values (v.e.v.) $h_{k}=\frac{1}{k}\left\langle\operatorname{Tr} \Phi^{k}\right\rangle$ of the scalar component $\Phi$ of $N=2$ gauge supermultiplet. Parameter $\tau$ is the ultraviolet (UV) bare complex coupling constant $\tau=\frac{4 \pi i}{e^{2}}+\frac{\theta}{2 \pi}$, which has direct physical meaning if the theory is $\mathrm{UV}$ finite. As soon as the gauge group is simple, there is only one gauge coupling constant $\tau$ in the UV.

The simplest example of an UV finite $4 d$ theory - on which we concentrate in what follows is the $N=2$ gauge theory with a matter hypermultiplet with mass $m$ in adjoint of the gauge group, which in the UV possesses $N=4$ supersymmetry and becomes conformally invariant. In the infrared (IR) limit the gauge group $G$ is generically broken by the v.e.v.'s $\langle\Phi\rangle$ down to $U(1)^{r_{G}}$. What survives are the $r_{G}$ light abelian gauge supermultiplets belonging to Cartan part of original supermultiplet in the adjoint of $G$. Their scalar components parametrize the flat valleys, and the corresponding background fields are refered to as $a_{k}$.

Exact effective action of this low-energy abelian theory is fully described by the "prepotential" $\left(N=2\right.$ superpotential) $\mathcal{F}\left(a_{k}\right)$, which of course depends non-trivially also on $h_{k}$ and $\tau$. One usualy introduces in addition to $a_{k}$ the "dual" variables $a_{k}^{D} \equiv \frac{\partial \mathcal{F}}{\partial a_{k}}$ and effective abelian charges $\mathcal{T}_{i j}=\frac{\partial a_{i}^{D}}{\partial a_{j}}=\frac{\partial^{2} \mathcal{F}}{\partial a_{i} \partial a_{j}}$. Moreover, whenever $m \neq 0$, at low energies a dynamical transmutation takes place and $\tau$ is substituted by the massive parameter ${ }^{2}$

$$
\Lambda_{\mathrm{QCD}}^{b_{1}}=\lim _{\substack{\tau \rightarrow i \infty \\ m \rightarrow \infty}} m^{b_{1}} e^{2 \pi i \tau}
$$

\footnotetext{
${ }^{2} b_{1}$ stands for the first (and unique in the case of a SUSY YM under consideration) coefficient of (Wilsonian) $\beta$-function, which in the $N=2$ theory is known to be equal to twice the dual Coxeter number of $G$ : $b_{1}=2 \mathrm{~h}_{G}^{\vee}$. One can make use of conformal invariance to set $m=1$, then the flow from $N=4$ to $N=2$ (i.e. from $m=0$ to $m=\infty$ ) is described as that to $\tau \rightarrow+i \infty$. According to [3] the moduli $h_{k}$ can be different in the UV (in the $N=4$ theory above $m$ ) and in the IR (in the $N=2$ one below $m$ ). This is obvious, once the moduli space in the IR has singularities at $\Lambda_{\mathrm{QCD}}$-dependent points, with $\Lambda_{\mathrm{QCD}}$ which itself depends on $\tau$ according to (2.1). The $\tau$-dependent difference between $h_{k}^{N=4}$ and $h_{k}^{N=2}$ is nicely described in the framework of integrability theory, see s.4.4 below.
} 
If the set of matter multiplets in the $N=2$ SUSY theory is not adjusted to make it UV finite, then $\Lambda_{\mathrm{QCD}}$ enters description of $\mathcal{C}\left(h_{k} \mid \Lambda_{\mathrm{QCD}}\right)$ instead of $\tau$.

Since both background fields $a_{k}$ and the v.e.v.'s $h_{k}$ are some coordinates along the valleys, they are related. Relation is non-trivial, because matrix elements (of which v.e.v. is an example) depends non-trivially on background fields. The main claim of $[2,3]$ is that this relation can be described by the formulas

$$
a_{i}=S_{A_{i}}=\oint_{A_{i}} d S, \quad a_{i}^{D}=S_{B_{i}}=\oint_{B_{i}} d S
$$

where $A_{i}$ and $B_{i}$ are conjugate $A$ and $B$-cycles on the curve $\mathcal{C} .^{3}$ What is not quite usual, in these theories the background fields $a_{i}, a_{i}^{D}$ are directly measurable. According to [17] they enter expression for the central charge of the $N=2$ superalgebra (central charges, as all the other Schwinger terms, are normally operator-valued beyond two dimensions), $Z=\sum_{i}\left(n_{i} a_{i}+n_{i}^{D} a_{i}^{D}\right)$, which defines the masses of BPS-saturated states in the small representations of superalgebra: $\mathcal{M}_{C}=2|Z|_{C}=2\left|\oint_{C} d S\right|$, with $C=\sum_{i}\left(n_{i} A_{i}+n_{i}^{D} B_{i}\right)$.

\section{Integrability theory}

According to [4]-[11] the picture, as described in the previous section, has exact counterpart in the theory of integrable systems $[18,19,20]$. On this side, instead of fixing a model of $N=2$ SUSY $Y M+$ matter, one inputs some integrable system. It is represented by the Lax operator $L(z \mid \tau)$, which depends on the complex "spectral parameter" $z$ - a coordinate on a complex "bare spectral curve", which is either a punctured Riemann sphere $S^{2}$ or torus (elliptic curve) $E(\tau)$. Then:

1) The entire spectral curve $\mathcal{C}\left(h_{k} \mid \tau\right)$ is described ${ }^{4}$ as a ramified covering over $E(\tau)$ :

$$
\operatorname{det}(L(z)-t)=0
$$

\footnotetext{
${ }^{3}$ In the " $4 d$ physical" context the possibility to represent explicitly (the cohomology class of) $d S$ depends largely on the adequate parametrization of the family $\mathcal{C}\left(h_{k}\right)$. For many of the $N=2$ theories this was achieved in refs. [12, $13,16,14,15]$ and nicely summarized in [5]. Sometime the number of $A$ and $B$ cycles on $\mathcal{C}$ exceeds $2 r_{G}$, however with the proper $d S$ all the extra integrals vanish due to the symmetries of the problem. Of course the adequate description arises automatically in the context of integrability theory: see $[4,5,8,10]$ and section 4 below.

${ }^{4}$ Let us remind that the main idea of algebro-geometrical approach to integrable equations [19] is to interpret the obvious "time"-invariance of eq.(3.2) (which follows immediately from the Lax equation, $\frac{\partial L}{\partial t_{\alpha}}=\left[L, A_{\alpha}\right], \quad A_{\alpha}=$ $\left.\mathcal{R}\left(\Delta^{\alpha-1} A\right)\right)$ as invariance of the (moduli of the) spectral curve $\mathcal{C}$ : moduli are integrals of motion. Clearly this statement (formulated as invariance of entire exact spectral surface of a theory under Hamiltonian flows) is not restricted to the field of conventional integrable systems (of KP/Toda family). Note that the curve, as defined in (3.2) depends also on representation $R$ of $G$ (since $L$ belongs to some $R$, not obligatory irreducible); in what follows we assume that $G$ labels the pair (Lie group, its representation).
} 
2) The $S_{C}$-variables are given by

$$
S_{C}=\oint_{C} t \omega
$$

In this picture $h_{k}$ (which were averages $\frac{1}{k}\left\langle\operatorname{Tr} \Phi^{k}\right\rangle$ in $4 d$ ) are the values of Hamiltonians (integrals of motion) of the integrable system. $S_{C}$ are its action integrals, $\frac{\partial S_{C}}{\partial h_{k}}$ being the (complex) periods of motion along the closed trajectories. Finally, $\omega(z)$ is canonical holomorphic 1-differential on $E(\tau)$ ("canonical" means that $\oint_{\mathcal{A}} \omega=1$, then $\left.\oint_{\mathcal{B}} \omega=\tau\right)$. As $\tau \rightarrow i \infty, E(\tau)$ degenerates into Riemann sphere $S_{(2)}^{2}$ with two punctures (conventionally placed at 0 and $\infty$ ), while $\omega$ acquires first-order poles at punctures with the residues $\oint_{ \pm \mathcal{A}} \omega= \pm 1$, i.e. $\omega \rightarrow \frac{1}{2 \pi i} \frac{d z}{z}$, and eq.(3.3) turns into

$$
S_{C} \stackrel{\tau \rightarrow i \infty}{\longrightarrow} \frac{1}{2 \pi i} \oint_{C} t d \log z
$$

It is a certain $\tau$-dependent combination of the Hamiltonians $h_{k}\left(h_{k}^{N=4}\right)$, that has a smooth limit (to be identified as $h_{k}^{N=2}$ ) as $\tau \rightarrow i \infty$.

According to [4] the "time"-flows of integrable theory should be interpreted as renormalization flows (dependence on the bare UV couplings) in original "target-space" $(4 d)$ model. The "time"-independence of the Hamiltonians, $\frac{\partial h_{k}}{\partial t_{\alpha}}=0$, is then nothing but renorminvariance of physicaly sensible v.e.v. $h_{k}$. Their $\left(h_{k}\right)$ dependence on the low-energy effective coupling constants (which is of course non-trivial) is then described in terms of effective low-energy Witham dynamics [21], deducible from dynamics of original integrable system. In certain sense the Whitham procedure can be considered as quantization of original (integrable) system around non-trivial background solutions (sometime the whole approach is called non-linear WKB). Such view is usefull for establishing connections to quantum groups and Langlands duality.

After the Whitham "slow" time-variables $T_{\alpha}$ are introduced, the prepotential $\mathcal{F}\left(a_{i}, T_{\alpha}\right)$ is identified as a "quasiclassical $\tau$-function", familiar to many from the studies of topological field theories $[22,23,24]$ and matrix models [25]. $\mathcal{F}$ is quadratic function of $a_{i}$ and $T_{\alpha}$, with coefficients made from the moduli $h_{k}$. However, $h_{k}$ are themselves depending on $a_{i}$ and $T_{\alpha}$, what makes $\mathcal{F}$ a highly non-trivial function. Still, as any quasiclassical $\tau$-function, it is homogeneous of degree 2 (though not just quadratic),

$$
\left(\sum_{i=1}^{r_{G}} a_{i} \frac{\partial}{\partial a_{i}}+\sum_{\alpha=0}^{\infty} T_{\alpha} \frac{\partial}{\partial T_{\alpha}}\right) \mathcal{F}=2 \mathcal{F} .
$$

Within the logic of [4] this condition is nothing but immediate corollary of scale invariance (a part of renorminvariance) of the v.e.v. $h_{k}$,

$$
\left(\sum_{i=1}^{r_{G}} a_{i} \frac{\partial}{\partial a_{i}}+\sum_{\alpha=0}^{\infty} T_{\alpha} \frac{\partial}{\partial T_{\alpha}}\right) h_{k}=0 .
$$


Of course, $\mathcal{F}$ is not homogeneous as a function of $a_{i}$ 's alone, because the coupling constants, not only background fields, are scale-dependent (whenever some $\beta$-functions are non-vanishing) - see $[6],[7]$ and [26] for detailed discussion of this point (for relation to a more sophisticated subject of Picard equations on the moduli space of spectral curves see [27]). The theory of prepotential and quasiclassical $\tau$-functions is a separate big issue (relevant far beyond $N=2$ SUSY YM) and will be discussed elsewhere. See [6] for a nice introduction and some references.

\section{Examples}

In the remaining part of these notes we demonstrate a little more explicitly how eqs.(3.2) and (3.3) for Calogero family of integrable systems can be used to reproduce the description of refs. [3] and [8] of the $N=4 \longrightarrow N=2$ flow. This can be helpfull to complement the presentation of refs. $[4,5,8,9,10] .{ }^{5}$

\subsection{Free particles (classical module space)}

Let us begin from the case when integrable system is just that of free particles. This example will be also used to fix the notation. Degrees of freedom are coordinates $\vec{q}=\left\{q_{i}\right\}$ and momenta $\vec{p}=\left\{p_{i}\right\}$. The bare spectral surface (where the spectral parameter $z$ originally takes values) is Riemann sphere $S_{(2)}^{2}$. In order to write down the Lax operator, let us denote the generators of $G$, associated with Cartan subalgebra and with the roots $\pm \vec{\alpha}$ through $\vec{H}$ and $E_{ \pm \vec{\alpha}}$ respectively. The "affine root" will be denoted $\vec{\alpha}_{0}$.

The Lax operator (familiar for many from the Drinfeld-Sokolov construction) is

$$
L_{\text {free }}(z)=\vec{p} \vec{H}+\sum_{\text {simple } \vec{\alpha}>\overrightarrow{0}} E_{\vec{\alpha}}+z E_{\vec{\alpha}_{0}}
$$

\footnotetext{
${ }^{5}$ Some of the explicit formulas below are given only for the fundamental representation of $G L(N)$, generalization to other cases is always straightforward. According to [5], the adequate description of $4 d$ gauge theory with non-simply laced gauge group $G$ is in terms of integrable system, associated with the dual group $G^{\vee}$.
} 
In the fundamental representation of $G L(n)$

$$
L_{\text {free }}^{f}(z)=\left(\begin{array}{cccccc}
p_{1} & 1 & 0 & & 0 & 0 \\
0 & p_{2} & 1 & & 0 & 0 \\
0 & 0 & p_{3} & \ldots & 0 & 0 \\
& & \cdots & & & \\
0 & 0 & 0 & & p_{n-1} & 1 \\
z & 0 & 0 & & 0 & p_{n}
\end{array}\right)
$$

The Hamiltonians (integrals of motion) are

$$
h_{k}=h_{k}^{(0)} \equiv \frac{1}{k} \sum_{i=1}^{n} p_{i}^{k}
$$

The full spectral curve $\mathcal{C}_{\text {free }}\left(h_{k}\right)$ is given by $(3.2)$ :

$$
\mathcal{C}_{\text {free }}\left(h_{k}\right): \quad \operatorname{det}\left(t-L_{\text {free }}(z)\right)=0,
$$

or, for the fundamental representation of $G L(n)$,

$$
\prod_{i=1}^{n}\left(t-p_{i}\right)=z
$$

In other words, $p_{i}$ are coordinates on the "classical module space" in the terminology of $[2,3]$.

Now we spend a paragraph for a play with notation. Let us denote

$$
\left.\hat{P}_{G}(t \mid h) \equiv \operatorname{det}\left(1-t^{-1} L_{\text {free }}\right)\right|_{z=0} .
$$

Since in $4 d$ picture $h_{k}=\frac{1}{k}\left\langle\operatorname{Tr} \Phi^{k}\right\rangle$, this can be also defined as

$$
\hat{P}_{G}(t \mid h)=\left\langle\operatorname{det}\left(1-t^{-1} \Phi\right)\right\rangle .
$$

$\hat{P}_{G}$ is obviously a polynomial in $t^{-1}$,

$$
\hat{P}_{G}(t \mid h)=\sum_{k} s_{k}^{G}(h) t^{-k}
$$

its coefficients being Schur polinomials of $h$ 's. In the case of fundamental representation of $G L(n)$ which we use in all the illustrative examples - it is more convenient to deal with $P_{n}(t)=t^{n} \hat{P}(t)=$ $\left.\operatorname{det}\left(t-L_{\text {free }}\right)\right|_{z=0}=\langle\operatorname{det}(t-\Phi)\rangle$. Thus

$$
P_{n}(t \mid h)=\sum_{k=0}^{n} s_{k}^{f}(h) t^{n-k}
$$


where the fundamental Schur polinomials are defined by $e^{\left(-\sum_{k=0}^{\infty} h_{k} t^{-k}\right)}=\sum_{k=0} s_{k}^{f}(h) t^{-k}$. (Note, that while $h_{k} \neq 0$ even for $k>n$ they depend algebraically on the first $n$ ones, $h_{1}, \ldots, h_{n}$. For such set of $\left\{h_{k}\right\}$, all the Schur polinomials $s_{k>n}^{f}(h)=0$. Their other characteristic property is $\frac{\partial s_{k}^{f}(h)}{\partial h_{l}}=s_{k-l}^{f}(h)$.)

In the new notation, the curve $\mathcal{C}_{\text {free }}(h)$ for the case of $G L(n)$ becomes

$$
\mathcal{C}_{\text {free }}\left(h_{k}\right): \quad P_{n}(t \mid h)=z
$$

with $P_{n}$ given by (4.6). For generic integrable systems, there is no such spliting between $t$ and $z$ variables: instead one can express eq.(3.2) through a more sophisticated $t$-polinomial

$$
\mathcal{P}_{n}(t|z| h)=\sum_{k=0}^{n} s_{k}^{f}(h) \mathcal{T}_{n-k}(t \mid z),
$$

where $\mathcal{T}_{k}(t \mid z)$ are still $k$-th order polinomials in $t$, but not just $t^{k}-z \delta_{k n}$ as in (4.7): they depend non-trivially on the spectral parameter $z .^{6}$

To complete our discussion of the free particle model, let us mention that since $\frac{1}{2 \pi i} t d \log z=$ $\sum_{i=1}^{n} \frac{t}{t-p_{i}} \frac{d t}{2 \pi i}$, the integrals $S_{C}$ are just linear combinations of $p_{i}$. Since one does not expect any quantum corrections, this is a right answer: $a_{i}$ just coincide with the moduli $p_{i}$. This is also in agreement with the quasiclassical interpretation of (3.3): for a free particle the normalized action integral along a closed trajectory of the length $l$ is $S=\frac{1}{l} \oint p d q=\frac{1}{l} \oint p \dot{q} d t=\frac{p^{2} T}{m l}$, while the period is $T=\frac{l}{\dot{q}}=\frac{l m}{p}$, so that $S=p$.

\subsection{Toda-chain system (quantum module space in the absence of matter)}

The bare spectral curve for this model is still $S_{(2)}^{2}$, and the Toda-chain Lax operator, associated with the algebra $G$ is given by [18]:

$$
L_{\mathrm{TC}}=\vec{p} \vec{H}+\sum_{\text {simple } \vec{\alpha}>\overrightarrow{0}}\left(E_{\vec{\alpha}}+e^{\vec{\alpha} \vec{q}} E_{-\vec{\alpha}}\right)+z E_{\vec{\alpha}_{0}}+\frac{1}{z} e^{\vec{\alpha}_{0} \vec{q}} E_{-\vec{\alpha}_{0}} .
$$

In the fundamental representation of $G L(n)$ the roots are represented as matrices $E_{i j}$ with non-vanishing entries at the crossing of $i$-th row and $j$-th column. For positive roots $i<j$ (upper tringular matrices), for negative roots $i>j$. Diagonal matrices represent Cartan elements. The

\footnotetext{
${ }^{6}$ Examples of this phenomenon below will include rational and elliptic Calogero systems, for which the bare spectral curve is $S_{(1)}^{2}$ and $E(\tau)$ respectively. Naturally, in the latter case the polinomials of Donagi and Witten [8] are reproduced (or rather linear combinations of those, which we believe are more appropriate, see s.4.3.2 below).
} 
simple positive/negative roots belong to the first upper/lower subdiagonal, the affine roots $\pm \vec{\alpha}_{0}$ are located at the left lower/ right upper corner respectively. Thus

$$
L_{\mathrm{TC}}^{f}(z)=\left(\begin{array}{cccccc}
p_{1} & 1 & 0 & 0 & \frac{1}{z} e^{q_{1}-q_{n}} \\
e^{q_{2}-q_{1}} & p_{2} & 1 & & 0 & 0 \\
0 & e^{q_{3}-q_{2}} & p_{3} & \cdots & 0 & 0 \\
& & \cdots & & & \\
0 & 0 & 0 & p_{n-1} & 1 \\
z & 0 & 0 & e^{q_{n}-q_{n-1}} & p_{n}
\end{array}\right)
$$

As soon as $z^{-1} \neq 0$, the system is periodic Toda chain, i.e. one can write $q_{n+1} \equiv q_{1}$. The first Hamiltonians (integrals of motion) are

$$
\begin{gathered}
h_{1}=\sum_{i=1}^{n} p_{i} \\
h_{2}=\sum_{i=1}^{n}\left(\frac{1}{2} p_{i}^{2}+e^{q_{i+1}-q_{i}}\right)=h_{2}^{(0)}+\sum_{i=1}^{n} e^{q_{i+1}-q_{i}},
\end{gathered}
$$

If one consdiers $S L(n)$ group instead of $G L(n)$, the first Hamiltonian $h_{1}=0$. Looking at the second Hamiltonian one easily recognizes the simplest equation of motion for the Toda chain:

$$
\frac{\partial^{2} q_{i}}{\partial t_{2}^{2}}=e^{q_{i+1}-q_{i}}-e^{q_{i}-q_{i-1}} .
$$

It is now an easy calculation to find the curve $\mathcal{C}_{\mathrm{TC}}\left(h_{k}\right)$ from eq.(3.2):

$$
\begin{gathered}
0=\operatorname{det}\left(t-L_{\mathrm{TC}}^{f}(z)\right)= \\
=t^{n}-t^{n-1}\left(\sum_{i=1}^{n} p_{i}\right)+t^{n-2}\left(\sum_{i<j}^{n} p_{i} p_{j}-\sum_{i=1}^{n} e^{q_{i+1}-q_{i}}\right)+\ldots-z-\frac{1}{z}= \\
=t^{n}-h_{1} t^{n-1}+\left(\frac{h_{1}^{2}}{2}-h_{2}\right) t^{n-2}+\ldots-z-\frac{1}{z}= \\
=P_{n}(t \mid h)-\left(z+\frac{1}{z}\right),
\end{gathered}
$$

where $P_{n}(t \mid h)$ is just the polinomial (4.6) from the previous section. There are only two differences from the free-particle case: First, the coefficients of this $P_{n}(t)$ are now Schur polinomials of Todachain Hamiltonians $h_{k}$, not just of the free ones $h_{k}^{(0)}$ - but this has no effect on the shape of the curve $\mathcal{C}$. Second - and this is the only trace of the switch from one integrable system to another is that instead of (4.7) we now get

$$
\mathcal{C}_{T C}(h): \quad P_{n}(t \mid h)=z+\frac{1}{z}
$$


with just the same polinomial $P_{n}(t \mid h)$.

A few comments are in order before we end this section. By a change of variables $z-\frac{1}{z}=Y$, eq.(4.12) can be transformed to the form

$$
Y^{2}-\left(P_{n}(t \mid h)\right)^{2}=\frac{1}{4}
$$

which was suggested for description of the quantum module space for the $N=2$ gauge theory without matter in refs. $[12,13,16]$. Since now $z=\frac{1}{2}\left(Y+P_{n}(t \mid h)\right)$, the integrals

$$
2 \pi i S_{C}=\oint_{C} t d \log z=\oint_{C} t d \log \left(Y+P_{n}(t \mid h)\right),
$$

in agreement with $[12,13,16]$.

In the simplest case of $G=S L(2)$, an obvious substitution $z=-e^{i \varphi} \operatorname{describes} \mathcal{C}_{T C}$ as $t^{2}-h^{2}+$ $2 \cos \varphi=0$, and

$$
2 \pi S_{C}=\oint_{C} t d \varphi=\oint_{C} \sqrt{h_{2}-2 \cos \varphi} d \varphi
$$

where the two integrals with $C=A$ and $C=B$ are over allowed and forbidden zones of classical motion in the sine-Gordon potential. This expression is the most straightforward illustration for the quasiclassical nature of (3.3), if considered from the point of view of integrability theory (while for $4 d$ physics this is a low-energy Bogolubov-Whitham limit).

Eq.(4.12) can be also represented as a system

$$
\begin{gathered}
z_{+}+z_{-}=P_{n}(t \mid h), \\
z_{+} z_{-}=1
\end{gathered}
$$

The spectral curve $\mathcal{C}_{\mathrm{TC}}$ is obtained by factorization w.r.to $Z_{2}$ transformation $z_{+} \leftrightarrow z_{-}$. The singular

(orbifold) points $z_{+}= \pm 1$ are exactly the two punctures of the bare spectral surface $S_{(2)}^{2}$. These orbifold points are blowed up in transition to $E(\tau)$ and $\mathcal{C}\left(h_{k} \mid \tau\right)$

\subsection{Calogero system}

\subsubsection{Elliptic Calogero (quantum moduli space in the UV-finite case)}

Expression (4.9) for the Toda-chain Lax operator looks a little artificial from the algebraic point of view, because it gives a special role to the simple roots. Clearly, its "more symmetric" counterpart exists, and is known as Calogero system [18] (its full name would be the Sutherland-Calogero-Moser-Olshanetsky- Perelomov-Ruijsenaars system). The Lax operator is

$$
L(z)=\vec{p} \vec{H}+\sum_{\vec{\alpha}} F(\vec{q} \vec{\alpha} \mid z) E_{\vec{\alpha}},
$$


where the sum goes over all (positive and negative) roots of $G$. Generically the bare spectral curve is elliptic, $E(\tau)$, and the function $F(q \mid z)$ is certain elliptic function of $z$ (it actually is not quite a double periodic function, but belongs to a linear bundle over $E(\tau))$. Its explicit appearence depends on parametrization of $E(\tau)$ : in varience with the Riemann sphere, elliptic curve has at least two widely useful parametrizations, to be refered to as "flat" and "elliptic". In the flat parametrization $E(\tau)$ is represented as a parallelogramm with the sides 1 and $\tau$, and we denote the spectral parameter in this parametrization through $\xi$ (leaving notation $z$ for parameter on $S^{2}$, which arises in degenerations of $E(\tau)$ ). Elliptic parametrization represents the same $E(\tau)$ as a double covering over Riemann sphere, $y^{2}=\left(x-e_{1}(\tau)\right)\left(x-e_{2}(\tau)\right)\left(x-e_{3}(\tau)\right)$.

Peculiar for the flat parametrization is a family of Weierstrass elliptic functions, which can be all constructed from theta-functions and represented as infinite sums or products. The double periodic Weierstrass function per se,

$$
\wp(\xi)=\frac{1}{\xi^{2}}+\sum_{\substack{-\infty<N, M<\infty \\ N^{2}+M^{2} \neq 0}}\left(\frac{1}{\left(\xi+N w_{1}+M w_{2}\right)^{2}}-\frac{1}{\left(N w_{1}+M w_{2}\right)^{2}}\right), \quad \tau=\frac{w_{2}}{w_{1}},
$$

is even and has double pole at $\xi=0$. We also need its integrals, $\zeta(\xi)$ and $\sigma(\xi): \wp(\xi)=-\frac{d \zeta(\xi)}{d \xi}=$ $-\frac{d^{2} \log \sigma(\xi)}{d \xi^{2}}$. They are not double periodic (but acquire non-trivial phase factors under the shifts $\left.\xi \rightarrow \xi+w_{l}, l=1,2\right)$, both are odd, $\zeta$ has simple pole, and $\sigma$ - simple zero at $\xi=0$. Further details can be found in any textbook on elliptic functions [28].

Generic Calogero function $F(q \mid \xi)$ is expressed through $\zeta$ and $\sigma$ [18]:

$$
F(q \mid \xi)=g \frac{\sigma(q-\xi)}{\sigma(q) \sigma(\xi)} e^{q \zeta(\xi)},
$$

It is double periodic in $\xi$, but not in $q$. Instead

$$
F\left(q \pm w_{l} \mid \xi\right)=F(q \mid \xi) e^{ \pm\left(w_{l} \zeta(\xi)-\zeta\left(w_{l}\right) \xi\right)} .
$$

Since exponential factor is independent of $q$, this transformation rule immediately implies that any product of the form

$$
F_{i_{1} i_{2}} F_{i_{2} i_{3}} \ldots F_{i_{k} i_{1}}
$$

with $F_{i j} \equiv F\left(q_{i j} \mid \xi\right), q_{i j}=q_{i}-q_{j}$ is double periodic in all the $q_{i}$ 's and $\xi$. In particular,

$$
F(q \mid \xi) F(-q \mid \xi)=g^{2}(\wp(\xi)-\wp(q)) .
$$

Further simplifications arise after symmetrization over indices $i_{1}, \ldots, i_{r}$. Introduce for $i_{1}<i_{2}<$ $\ldots<i_{r}$

$$
\left(\mathcal{S}^{r} F\right)_{i_{1} \ldots i_{r}} \equiv \frac{1}{r} \sum_{\substack{\text { all permutations of } \\ i_{1} \ldots i_{r}}} F_{i_{1} i_{2}} F_{i_{2} i_{3}} \ldots F_{i_{k} i_{1}} .
$$


Every item in the sum has two simple poles in every argument $q_{i_{s}}$ (so that $\left(\mathcal{S}^{r} F\right)$ could have simple poles whenever $q_{i_{s}}=q_{i_{s^{\prime}}}$ ). It is, however, easy to check that the residue at the pole cancels completely between different items in the sum (4.17) as $r>2$. Thus, as a function of any $q_{i_{s}}$ our $\left(\mathcal{S}^{r} F\right)$ has no singularities - therefore it does not depend on any of $q_{i_{s}}$ 's at all. This means that the indices $i_{1} \ldots i_{r}$ can be also omited at the l.h.s. of (4.17), and $\left(\mathcal{S}^{r} F\right)_{i_{1} \ldots i_{r}}=\left(\mathcal{S}^{r} F\right)(\xi)$ is a function of $\xi$ only. This function can be easily evaluated (for example, by evaluating the sum on the r.h.s. of (4.17) at some special values of $q_{i_{s}}$, like $q_{k}=k \xi$ ):

$$
\left(\mathcal{S}^{r} F\right)(\xi)=(-g)^{r}\left(\frac{d}{d \xi}\right)^{r-2} \wp(\xi), \quad r>2 .
$$

(Asymptotical behaviour, $\left(\mathcal{S}^{r} F\right)(\xi)=\frac{(r-1) !}{\xi^{r}}(1+o(\xi))$ is very simple to reproduce in the rational limit of Calogero system, see s.4.3.3 below.)

We now return to Calogero system and restrict to our usual example of fundamental representation of $G L(n)$. The first Hamiltonians of Calogero system are:

$$
\begin{gathered}
h_{1}=\sum_{i=1}^{n} p_{i}, \\
h_{2}=\frac{1}{2} \sum_{i=1}^{n} p_{i}^{2}-g^{2} \sum_{i<j} \wp\left(q_{i}-q_{j} \mid \xi\right)=h_{2}^{(0)}-g^{2} \sum_{i<j} \wp\left(q_{i j} \mid \xi\right), \\
h_{3}=h_{3}^{(0)}-g^{2} \sum_{i \neq j} p_{i} \wp\left(q_{i j} \mid \xi\right), \ldots
\end{gathered}
$$

(see (4.5) for the definition of $\left.h_{k}^{(0)}\right)$. The Lax operator

$$
L(\xi)=\left(\begin{array}{ccccc}
p_{1} & F_{12} & F_{13} & & F_{1 n} \\
F_{21} & p_{2} & F_{23} & \ldots & F_{2 n} \\
& & \ldots & & \\
F_{n 1} & F_{n 2} & F_{n 3} & & p_{n}
\end{array}\right)
$$

(Note that there is no special role assigned to the affine roots: they appear automatically in appropriate degeneration limit $E(\tau) \rightarrow S_{(2)}^{2}$, see s.4.4 below.) Eq.(3.3) is now:

$$
\mathcal{C}\left(h_{k} \mid \tau\right): \quad \mathcal{P}_{n}(t|h| \xi)=0
$$


with

$$
\begin{gathered}
\mathcal{P}_{n}(t|h| \xi) \equiv \operatorname{det}\left(t-L^{f}(\xi)\right)= \\
=t^{n}-t^{n-1} \sum_{i=1}^{n} p_{i}+t^{n-2} \sum_{i<j}^{n}\left(p_{i} p_{j}-F_{i j} F_{j i}\right)- \\
-t^{n-3} \sum_{i<j<k}^{n}\left(p_{i} p_{j} p_{k}-p_{i} F_{j k} F_{k j}-p_{j} F_{i k} F_{k i}-p_{k} F_{i j} F_{j i}+F_{i j} F_{j k} F_{k i}+F_{i k} F_{k j} F_{j i}\right)+ \\
+\ldots
\end{gathered}
$$

The first few terms on the r.h.s. are easily evaluated with the help of (4.16) and explicit expressions (4.19) for the first few $h_{k}$. The first two are just $t^{n}-h_{1} t^{n-1}$ (of course $h_{1}^{(0)}=h_{1}$ ). Further:

The $t^{n-2}$ term:

$$
\begin{gathered}
\sum_{i<j} p_{i} p_{j}=\frac{1}{2}\left(\left(\sum_{i=1}^{n} p_{i}\right)^{2}-\sum_{i=1}^{n} p_{i}^{2}\right)=\frac{h_{1}^{2}}{2}-h_{2}^{(0)} \\
-\sum_{i<j} F_{i j} F_{j i} \stackrel{(4.16)}{=}-\frac{n(n-1)}{2} g^{2} \wp(\xi)+g^{2} \sum_{i<j} \wp\left(q_{i j}\right) .
\end{gathered}
$$

Together, the last items of the two expressions combine into the full Hamiltonian $-h_{2}$, and we get:

$$
\left(\frac{h_{1}^{2}}{2}-h_{2}-\frac{n(n-1)}{2} g^{2} \wp(\xi)\right) t^{n-2}
$$

The $t^{n-3}$ term:

$$
\begin{gathered}
\sum_{i<j<k} p_{i} p_{j} p_{k}=\frac{h_{1}^{3}}{6}-h_{1} h_{2}^{(0)}+h_{3}^{(0)} \\
-\sum_{i<j<k}\left(p_{i} F_{j k} F_{k j}+p_{j} F_{i k} F_{k i}+p_{k} F_{i j} F_{j i}\right)= \\
\stackrel{(4.16)}{=}-\frac{n(n-1)}{2} g^{2} \wp(\xi)\left(\sum_{i=1}^{n} p_{i}\right)+g^{2} \sum_{i<j<k}\left(p_{i} \wp\left(q_{j k}\right)+p_{j} \wp\left(q_{i k}\right)+p_{k} \wp\left(q_{i j}\right)\right)= \\
=-\frac{n(n-1)}{2} g^{2} \wp(\xi) h_{1}+g^{2}\left(\sum_{i=1}^{n} p_{i}\right)\left(\sum_{j<k} \wp\left(q_{j k}\right)\right)-g^{2} \sum_{i \neq j} p_{i} \wp\left(q_{i j}\right) .
\end{gathered}
$$

The two last terms serve to complement $h_{2}^{(0)}$ and $h_{3}^{(0)}$ till full $h_{2}$ and $h_{3}$. The remaining contribution to the $t^{n-3}$ term is just

$$
\sum_{i<j<k}\left(\mathcal{S}^{3} F\right)_{i j k} \stackrel{(4.18)}{=} \frac{n(n-1)(n-2)}{6}\left(\mathcal{S}^{3} F\right)(\xi)=-\frac{n(n-1)(n-2)}{6} g^{3} \wp^{\prime}(\xi) .
$$

Bringing things together we obtain for (4.21):

$$
\begin{gathered}
\mathcal{P}(t|h| \xi)=t^{n}-h_{1} t^{n-1}+\left(\frac{h_{1}^{2}}{2}-h_{2}-\frac{n(n-1)}{2} g^{2} \wp(\xi)\right) t^{n-2}+ \\
+\left(\frac{h_{1}^{3}}{6}-h_{1} h_{2}+h_{3}-\frac{n(n-1)}{2} g^{2} \wp(\xi) h_{1}-\frac{n(n-1)(n-2)}{6} g^{3} \wp^{\prime}(\xi)\right) t^{n-3}-\ldots
\end{gathered}
$$


It is reasonable to rearrange this sum, collecting terms with the same $h$-dependence (and thus in a sense separate $h$ - and $\tau$ dependencies - the latter one entering through Weierstrass functions):

$$
\begin{gathered}
\mathcal{P}_{n}(t|h| \xi)=\sum_{k=0} s_{k}^{f}(h) \mathcal{T}_{n-k}(t \mid \xi)= \\
=\mathcal{T}_{n}(t \mid \xi)-h_{1} \mathcal{T}_{n-1}(t \mid \xi)+\left(\frac{h_{1}^{2}}{2}-h_{2}\right) \mathcal{T}_{n-2}(t \mid \xi)-\left(\frac{h_{1}^{3}}{6}-h_{1} h_{2}+h_{3}\right) \mathcal{T}_{n-3}(t \mid \xi)+\ldots
\end{gathered}
$$

The new $h$-independent $t$-polinomials here are:

$$
\mathcal{T}_{n}(t \mid \xi)=t^{n}-\frac{n(n-1)}{2} g^{2} \wp(\xi) t^{n-2}+\frac{n(n-1)(n-2)}{6} g^{3} \wp^{\prime}(\xi) t^{n-3}-\ldots
$$

Our next task is to derive a generic formula for $\mathcal{T}_{n}$.

The coefficient of $t^{n-\ell}$ in $\mathcal{T}_{n}$ comes from evaluation of the minor of the Lax operator at $p_{i}=0$,

$$
M_{\ell}=\left(\begin{array}{ccccc}
0 & F_{12} & F_{13} & & F_{1 \ell} \\
F_{21} & 0 & F_{23} & \ldots & F_{2 \ell} \\
& & \ldots & & \\
F_{\ell 1} & F_{\ell 2} & F_{\ell 3} & & 0
\end{array}\right)
$$

$M_{\ell}$ is obviously an algebraic combination of $\left(\mathcal{S}^{r} F\right)$ :

$$
\begin{gathered}
M_{2}=-\left(\mathcal{S}^{2} F\right)_{12}, \quad M_{3}=+\left(\mathcal{S}^{3} F\right)_{123} \\
M_{4}=\left(\mathcal{S}^{2} F\right)_{12}\left(\mathcal{S}^{2} F\right)_{34}+\left(\mathcal{S}^{2} F\right)_{13}\left(\mathcal{S}^{2} F\right)_{24}+\left(\mathcal{S}^{2} F\right)_{14}\left(\mathcal{S}^{2} F\right)_{23}-\left(\mathcal{S}^{4} F\right)_{1234}, \quad \ldots
\end{gathered}
$$

The $q$-dependent part of $M_{\ell}$ is always absorbed into the Hamiltonians $h_{k}$ and does not contribute to the coefficients of $h$-independent $\mathcal{T}_{n}$. Thus in the study of $\mathcal{T}_{n}$ we can neglect $q$-dependent contributions to $M_{\ell}$ and substitute $M_{\ell}(\{q\} ; \xi) \rightarrow \hat{M}_{\ell}(\xi)$. According to (4.18) the only source of $q$-dependence is $\left(\mathcal{S}^{2} F\right)$, thus for our purposes it is enough to drop the second item at the r.h.s. of (4.16), i.e. just use the same expression (4.18) for $r=2$. After this the matrix indices can be neglected, and we face a pure combinatorial problem of decomposing an integer $\ell$ into a sum of different integers $r_{s}$, every $r_{s}$ coming with multiplicity $m_{s}$. Thus

$$
\begin{gathered}
\hat{M}_{\ell}=(-)^{\ell} \sum_{\substack{2 \leq r_{1}<r_{2}<\ldots \\
m_{s}>0}} \frac{\ell !}{\prod_{s} m_{s} !\left(r_{s} !\right)^{m_{s}}} \prod_{s}\left(-\mathcal{S}^{r_{s}} F\right)^{m_{s}} . \\
\sum_{s} m_{s} r_{s}=\ell
\end{gathered}
$$

It remains to include the factor $\frac{n !}{\ell !(n-\ell) !}$ for the number of minors like $M_{\ell}$ in the $n \times n$ matrix, and substitute $\left(\mathcal{S}^{r} F\right)$ from $(4.18)$ in order to obtain:

$$
\begin{gathered}
t^{-n} \mathcal{T}_{n}(t \mid \xi)=1+ \\
+\sum_{\substack{2 \leq r_{1}<r_{2}<\ldots \\
m_{s}>0}} \frac{n !}{\left(n-\sum_{s} m_{s} r_{s}\right) !} \prod_{s} \frac{(-)^{m_{s}}}{m_{s} !\left(r_{s} !\right)^{m_{s}}}\left(-\frac{g}{t}\right)^{\sum_{s} m_{s} r_{s}} \prod_{s}\left(\partial_{\xi}^{r_{s}-2} \wp(\xi)\right)^{m_{s}}
\end{gathered}
$$


Obviously, $\frac{\partial \mathcal{T}_{n}}{\partial t}=n \mathcal{T}_{n-1}$ - what makes $\mathcal{T}_{n}(t)$ somewhat similar to Schur polinomials.

Equations (4.20), (4.24) and (4.25) provide a complete description of the full spectral curve $\mathcal{C}\left(h_{k} \mid \tau\right)$, induced by the flat parametrization of the bare spectral curve $E(\tau)$. It should be supplemented by eq.(3.3) for the action integrals. Since in the flat parametrization $\omega=\frac{1}{w_{1}} d \xi$,

$$
S_{C}=\frac{1}{w_{1}} \oint_{C} t d \xi
$$

One can easily convert to elliptic parametrization of $E(\tau)$, when instead of $\xi$ one uses $x, y$ : $y^{2}=\prod_{a=1}^{3}\left(x-e_{a}(\tau)\right)$. It is enough to recall that

$$
\left(\wp^{\prime}\right)^{2}(\xi)=4 \wp^{3}(\xi)-\mathrm{g}_{2}(\tau) \wp(\xi)-\mathrm{g}_{3}(\tau)=4 \prod_{a=1}^{3}\left(\wp(\xi)-e_{a}^{0}(\tau)\right)
$$

where $e_{1}^{0}+e_{2}^{0}+e_{3}^{0}=0$. Given $e_{a}(\tau)$ in generic position, $e_{a}^{0}=e_{a}-s, \quad s(\tau) \equiv \frac{1}{3} \sum_{a} e_{a}$. Thus we have identification:

$$
\begin{gathered}
x=\wp(\xi)+s(\tau), \\
y=\frac{1}{2} \wp^{\prime}(\xi) .
\end{gathered}
$$

Higher derivatives of $\wp$-function are algebraic functions of $\wp, \wp^{\prime}$, and thus of $x, y$ and $e_{a}$, for example:

$$
\begin{gathered}
\wp^{\prime \prime}(\xi)=6 \wp^{2}(\xi)-\frac{g_{2}}{2}=6(x-s)^{2}-\sum_{a=1}^{3}\left(e_{a}^{0}\right)^{2}=6 x^{2}-12 x s+9 s^{2}-\sum_{a=1}^{3} e_{a}^{2}, \\
\wp^{\prime \prime \prime}(\xi)=12 \wp \wp^{\prime}(\xi)=24(x-s) y,
\end{gathered}
$$

Substituting these expressions into (4.25) one obtains $\mathcal{T}_{n}(t \mid x, y)$ and thus the equation for the spectral curve $\mathcal{C}\left(h_{k} \mid \tau\right)$ in elliptic parametrization. Comparison with description of [3] (in the only available case of $S U(2))$ identifies the Calogero coupling $g$ with the mass of adjoint hypermultiplet: ${ }^{7}$

$$
g^{2}=\frac{m^{2}}{8}\left(\frac{i w_{1}}{\pi}\right)^{2}
$$

\footnotetext{
${ }^{7}$ For this, the curve $\mathcal{C}_{S U(2)}\left(h_{2} \mid \tau\right)$, defined by the pair of equations, $\mathcal{T}_{2}-h_{2} \mathcal{T}_{0}=t^{2}-g^{2}(x-s)-h_{2}=0$, and $y^{2}=\prod_{a=1}^{3}\left(x-e_{a}\right)$, or just $w^{2} \equiv\left(\frac{y t}{g}\right)^{2}=\left(x+\frac{h_{2}}{g^{2}}-s\right) \prod_{a=1}^{3}\left(x-e_{a}\right)$, should be compared with the one from [3], $w^{2}=\prod_{a=1}^{3}\left(x-2 \hat{e}_{a} h_{2}-\frac{1}{4} \hat{e}_{a}^{2} m^{2}\right)$. The argument of [3] requires $\hat{e}_{a}(\tau)$ to have certain modular weight and asymptotics $\hat{e}_{1}(\tau=i \infty)=\frac{2}{3}$, thus they are actually $\hat{e}_{a}=\left(\frac{w_{1}}{\pi}\right)^{2} e_{a}^{0}=\left(\frac{w_{1}}{\pi}\right)^{2}\left(e_{a}-s\right)$. The two curves are equivalent provided the double ratios coincide,

$$
\frac{g^{-2} h_{2}-s+e_{1}}{g^{-2} h_{2}-s+e_{2}} \cdot \frac{e_{3}-e_{2}}{e_{3}-e_{1}}=\frac{\left(e_{3}-e_{2}\right)\left(2 h_{2}+\left(e_{2}^{0}+e_{3}^{0}\right) \frac{m^{2}}{4}\left(\frac{w_{1}}{\pi}\right)^{2}\right)}{\left(e_{3}-e_{1}\right)\left(2 h_{2}+\left(e_{1}^{0}+e_{3}^{0}\right) \frac{m^{2}}{4}\left(\frac{w_{1}}{\pi}\right)^{2}\right)},
$$

i.e. $h_{2}-g^{2}\left(s-e_{1,2}\right)=h_{2}+\frac{m^{2}}{8}\left(\frac{w_{1}}{\pi}\right)^{2}\left(e_{2,1}^{0}+e_{3}^{0}\right)=h_{2}+\frac{m^{2}}{8}\left(\frac{w_{1}}{\pi}\right)^{2}\left(s-e_{1,2}\right)$, or $g=\frac{m}{2 \sqrt{2}} \frac{i w_{1}}{\pi}$. The $h_{2}$ here is by definition $h_{2}^{N=4}$.
} 
Finally, since $\omega=\frac{1}{w_{1}} d \xi=\frac{1}{w_{1}} \frac{d \wp(\xi)}{\wp^{\prime}(\xi)}=\frac{2}{w_{1}} \frac{d x}{y}$,

$$
S_{C}=\frac{2}{w_{1}} \oint_{C} \frac{t d x}{y} .
$$

Note, that in elliptic parametrization $w_{1}$ is not independent of $e_{a}(\tau)$, and can not be put equal to unity, what is always allowed in the flat parametrization. Instead, $w_{1}=2 \oint_{\mathcal{A}} \frac{d x}{y}$.

\subsubsection{Appendix. Comparison with Donagi-Witten polinomials}

Since there is no general formula for all the $\partial^{k} \wp(\xi)$ in elliptic parametrization, one can just evaluate every particulat $\mathcal{T}_{n}$ explicitly. For this we first need them in the flat parametrization. Allowed sets $\left\{r_{m}\right\}$ for the first few $n$ in (4.25) are:

$$
\begin{array}{lc}
n=0: & \emptyset \\
n=1: & \emptyset \\
n=2: & \left\{\left(2_{1}\right)\right\} \\
n=3: & \left\{\left(2_{1}\right),\left(3_{1}\right)\right\} \\
n=4: & \left\{\left(2_{1}\right),\left(3_{1}\right),\left(4_{1}\right),\left(2_{2}\right)\right\} \\
n=5: & \left\{\left(2_{1}\right),\left(3_{1}\right),\left(4_{1}\right),\left(2_{2}\right),\left(5_{1}\right),\left(2_{1}, 3_{1}\right)\right\}
\end{array}
$$

Given this, we read from (4.25):

$$
\begin{aligned}
& \mathcal{T}_{0}=1, \\
& \mathcal{T}_{1}=t \\
& \mathcal{T}_{2}=t^{2}-g^{2} \wp(\xi), \\
& \mathcal{T}_{3}=t^{3}-3 g^{2} \wp(\xi) t+g^{3} \wp^{\prime}(\xi), \\
& \mathcal{T}_{4}=t^{4}-6 g^{2} \wp(\xi) t^{2}+4 g^{3} \wp^{\prime}(\xi) t-g^{4}\left(\wp^{\prime \prime}(\xi)-3 \wp^{2}(\xi)\right), \\
& \mathcal{T}_{5}=t^{5}-10 g^{2} \wp(\xi) t^{3}+10 g^{3} \wp^{\prime}(\xi) t^{2}-5 g^{4}\left(\wp^{\prime \prime}(\xi)-3 \wp^{2}(\xi)\right)+g^{5}\left(\wp^{\prime \prime \prime}(\xi)-10 \wp \wp^{\prime}(\xi)\right),
\end{aligned}
$$


Next we omit $g$ (rescale $t \rightarrow g t$ and $\mathcal{T}_{n} \rightarrow g^{n} \mathcal{T}_{n}$ ) and substitute (4.26) and (4.27):

$$
\begin{aligned}
\mathcal{T}_{0}= & 1, \\
\mathcal{T}_{1}= & t \\
\mathcal{T}_{2}= & t^{2}-x+s \\
& \stackrel{s=0}{\Longrightarrow} t^{2}-x \\
\mathcal{T}_{3}= & t^{3}-3 x t+3 s t+2 y \\
& \stackrel{s=0}{\Longrightarrow} t^{3}-3 x t+2 y \\
\mathcal{T}_{4}= & t^{4}-6 x t^{2}+6 s t^{2}+8 y t-3 x^{2}+6 s x-6 s^{2}+3 s_{2} \\
& \stackrel{s=0}{\Longrightarrow} t^{4}-6 x t^{2}+8 y t-3 x^{2}+3 s_{2}, \\
\mathcal{T}_{5}= & t^{5}-10 x t^{3}+10 s t^{3}+20 y t^{2}-15 x^{2} t+30 s x t-30 s^{2} t+15 s_{2} t+4 x y-4 s y \\
& \stackrel{s=0}{\Longrightarrow} t^{5}-10 x t^{3}+20 y t^{2}-15 x^{2} t+15 s_{2} t+4 x y,
\end{aligned}
$$

Here $s=s_{1}(\tau) \equiv \frac{1}{3} \sum_{a=1}^{3} e_{a}, \quad s_{2}(\tau) \equiv \frac{1}{3} \sum_{a=1}^{3} e_{a}^{2}$.

These expressions can be now compared with Donagi-Witten polinomials [8]:

$$
\begin{array}{ll}
\mathcal{T}_{0}^{\mathrm{DW}}=1 & =\mathcal{T}_{0}, \\
\mathcal{T}_{1}^{\mathrm{DW}}=t & =\mathcal{T}_{1}, \\
\mathcal{T}_{2}^{\mathrm{DW}}=t^{2}-x & =\mathcal{T}_{2}-s \mathcal{T}_{0}, \\
\mathcal{T}_{3}^{\mathrm{DW}}=t^{3}-3 x t+2 y & =\mathcal{T}_{3}-3 s \mathcal{T}_{1}, \\
\mathcal{T}_{4}^{\mathrm{DW}}=t^{4}-6 x t^{2}+8 y t-3 x^{2}+12 s x & =\mathcal{T}_{4}-6 s \mathcal{T}_{2}+\left(12 s^{2}-3 s_{2}\right) \mathcal{T}_{0}, \\
\mathcal{T}_{5}^{\mathrm{DW}}=t^{5}-10 x t^{3}+20 y t^{2}-15 x^{2} t+ & \\
\quad+60 s x t-4 x y-24 s y & =\mathcal{T}_{5}-10 s \mathcal{T}_{3}+5\left(12 s^{2}-3 s_{2}\right) \mathcal{T}_{1},
\end{array}
$$

We see that Donagi-Witten polinomials are some linear combinations of $\mathcal{T}_{n}$, however, the linear transformation depends on $\tau$ and thus is not quite innocent. Moreover, this dependence does not disappear even for the natural choice $s=\frac{1}{3} \sum_{a} e_{a}(\tau)=0$, since $s_{2}(\tau)=\frac{1}{3} s^{2}-\frac{1}{6} \mathrm{~g}_{2}(\tau)$ can not be made vanishing by choice. Let us remind that $\mathcal{T}_{n}$ are defined in such a way that the equation for 
the curve is

$$
\mathcal{C}\left(h_{k} \mid \tau\right): \quad \sum_{k} s_{k}^{f}(h) \mathcal{T}_{n-k}=0,
$$

with $\tau$-independent $s_{k}^{f}(h)$ - i.e. the $h$-dependence is fully separated from $\tau$-dependence. This property will not be true for the basis of $\mathcal{T}_{n}^{\mathrm{DW}}$.

\subsubsection{Appendix. Rational Calogero (cusp singularity on the bare spectral curve)}

It is instructive to consider the simple "rational" limit of Calogero system, obtained when both periods of $E(\tau)$ become large: $w_{1}, w_{2} \longrightarrow \infty$. Then

$$
\begin{gathered}
F(q \mid \xi) \longrightarrow\left(\frac{1}{z}-\frac{1}{q}\right) e^{q / z} \\
\sigma(\xi) \longrightarrow z, \quad \zeta(\xi) \longrightarrow \frac{1}{z}, \quad \wp(\xi) \longrightarrow \frac{1}{z^{2}}, \quad \wp^{\prime}(\xi) \longrightarrow-\frac{2}{z^{3}},
\end{gathered}
$$

and the curve $E(\tau)$ degenerates into $y^{2}=x^{3}$.

Remarkably, the polinomials $\mathcal{T}_{n}(t)$ remain non-trivial in this limit: since

$$
\partial^{r_{s}-2} \wp(\xi) \longrightarrow(-)^{r_{s}} \frac{\left(r_{s}-1\right) !}{z^{r_{s}}},
$$

eq.(4.25) gives

$$
t^{-n} \mathcal{T}_{n}^{\mathrm{rat}}(t \mid z)=1+\sum_{\substack{2 \leq r_{1}<r_{2}<\ldots \\ m_{s}>0}} \frac{n !}{\left(n-\sum_{s} m_{s} r_{s}\right) !} \prod_{s} \frac{(-)_{s}^{m}}{m_{s} !\left(r_{s}\right)^{m_{s}}}\left(-\frac{g}{z t}\right)^{\sum_{s} m_{s} r_{s}}
$$

e.g. $\mathcal{T}_{0}^{\mathrm{rat}}=1, \mathcal{T}_{1}^{\mathrm{rat}}=t, \mathcal{T}_{2}^{\mathrm{rat}}=t^{2}-\frac{g^{2}}{z^{2}}, \mathcal{T}_{3}^{\mathrm{rat}}=t^{3}-\frac{3 g^{2} t}{z^{2}}+\frac{2 g^{3}}{z^{3}}, \ldots$

This limit can be also used to illustrate the derivation of (4.16) and (4.18). Indeed, it is just straightforward to check:

$$
F_{12}^{\mathrm{rat}} F_{21}^{\mathrm{rat}}=\left(\frac{1}{z}-\frac{1}{q_{12}}\right)\left(\frac{1}{z}-\frac{1}{q_{21}}\right)=\frac{1}{z^{2}}-\frac{1}{q_{12}^{2}}
$$

- in accordance with (4.16). Further,

$$
\begin{gathered}
F_{12}^{\mathrm{rat}} F_{23}^{\mathrm{rat}} F_{31}^{\mathrm{rat}}=\left(\frac{1}{z}-\frac{1}{q_{12}}\right)\left(\frac{1}{z}-\frac{1}{q_{23}}\right)\left(\frac{1}{z}-\frac{1}{q_{31}}\right)= \\
=\frac{1}{z^{3}}-\frac{1}{z^{2}}\left(\frac{1}{q_{12}}+\frac{1}{q_{23}}+\frac{1}{q_{31}}\right)-\frac{1}{q_{12} q_{23} q_{31}} .
\end{gathered}
$$

Note that the term with $\frac{1}{z}$ vanishes. Because of this the symmetric combination

$$
\left(\mathcal{S}^{3} F^{\mathrm{rat}}\right)_{123} \equiv F_{12}^{\mathrm{rat}} F_{23}^{\mathrm{rat}} F_{31}^{\mathrm{rat}}+F_{13}^{\mathrm{rat}} F_{32}^{\mathrm{rat}} F_{21}^{\mathrm{rat}}=\frac{2}{z^{3}} \longleftarrow-\wp^{\prime}(\xi)
$$


in accordance with (4.18). The idea of the general proof of (4.18) can be also illustrated with this example. Consider the residue at pole $q_{12}=0$. In case of $F_{12}^{\mathrm{rat}} F_{23}^{\mathrm{rat}} F_{31}^{\mathrm{rat}}$ it is equal to $-\left.F_{23}^{\mathrm{rat}} F_{31}^{\mathrm{rat}}\right|_{1=2}=$ $-F_{23}^{\text {rat }} F_{32}^{\text {rat }}$, while in the case of $F_{13}^{\text {rat }} F_{32}^{\text {rat }} F_{21}^{\text {rat }}$ it is rather $+\left.F_{13}^{\text {rat }} F_{32}^{\text {rat }}\right|_{1=2}=+F_{23}^{\text {rat }} F_{32}^{\text {rat }}$. Thus this pole disappears from $\left(\mathcal{S}^{3} F\right)$, as well as the other would be poles at $q_{23}=0$ and $q_{13}=0$. We conclude that $\left(\mathcal{S}^{3} F\right)$ does not depend of $q$ 's. The reasoning is just the same for all $\left(\mathcal{S}^{r} F\right)$, as $r>2$ they all are $q$-independent, and their $z$-dependence is not difficult to work out:

$$
\left(\mathcal{S}^{r} F^{\mathrm{rat}}\right)(z)=\frac{(r-1) !}{z^{r}} \longleftarrow\left(-\frac{d}{d \xi}\right)^{r-2} \wp(\xi), \quad \text { for } r>2 .
$$

\subsection{From elliptic Calogero (gauge $N=4$ ) to Toda-chain (gauge $N=2$ without matter)}

Of more interest are asymmetric limits of Calogero system, when the ratio of periods $\tau=\frac{w_{2}}{w_{1}} \rightarrow i \infty$ (see [29] and references therein). Since $w_{1}$ is finite it is convenient to fix it, the natural choice being $w_{1}=-i \pi\left(\right.$ thus $\left.w_{2}=w_{1} \tau=-i \pi \tau\right)$. Then

$$
\begin{gathered}
\wp(\xi)=\left(\frac{\pi}{w_{1}}\right)^{2}\left(\sum_{-\infty<M<\infty} \frac{1}{\sin ^{2} \frac{\pi}{w_{1}}\left(\xi+M w_{2}\right)}-C(\tau)\right) \stackrel{w_{1}=-i \pi}{=} \\
=\sum_{-\infty<M<\infty} \frac{1}{\sinh ^{2}\left(\xi+M w_{2}\right)}+C(\tau),
\end{gathered}
$$

where

$$
\begin{aligned}
C(\tau)=\frac{1}{3} & +2 \sum_{M \geq 1} \frac{1}{\sin ^{2} \frac{\pi}{w_{1}} M w_{2}}=\frac{1}{3}+2 \sum_{M \geq 1} \frac{1}{\sin ^{2} \pi M \tau}= \\
& =\frac{1}{3}\left(1-24 \sum_{M \geq 1} \frac{e^{2 \pi i M \tau}}{\left(1-e^{2 \pi i M \tau}\right)^{2}}\right) .
\end{aligned}
$$

If now $\tau \rightarrow i \infty\left(w_{2} \rightarrow+\infty\right)$ and $\xi$ is kept finite, there is only one term surviving in the sum: the one with $M=0: \wp(\xi) \rightarrow(\sinh \xi)^{-2}$. This gives trigonometric Calogero system with potential

$$
V^{\operatorname{tri}}(q)=-g^{2} \sum_{j<k}^{n} \frac{1}{\sinh ^{2} q_{j k}} .
$$

Next, one can consider a scaling limit, when coupling constant $g^{2}$ grows along with the growth of $|\tau|: g=i g_{0} e^{\kappa w_{2}}=i g_{0} e^{-i \kappa \pi \tau}$. In order to keep $V^{\text {tri }}$ finite one should require that all $\operatorname{Re} q_{j k} \geq$ $\kappa w_{2}+O(1)\left(\right.$ since $(\sinh q)^{-2}=e^{-2 q}\left(1+O\left(e^{-2 q}\right)\right)$. This condition can not be saturated for all the pairs $(j, k)$ at once, because $q_{j l}=q_{j k}+q_{k l}$. The only option is to take

$$
q_{j k}=\kappa w_{2}(k-j)+\frac{1}{2} \hat{q}_{j k} \quad \text { for } j<k
$$


(i.e. $q_{j}=-j \kappa w_{2}+\frac{1}{2} \hat{q}_{j}$ with already finite $\hat{q}_{j}$ ). Then only terms with $|j-k|=1$ will contribute to $V^{\text {tri }}$ in the limit $w_{2} \rightarrow+\infty$ :

$$
\begin{gathered}
V_{0}^{\mathrm{TC}}(\hat{q})=\lim _{\tau \rightarrow i \infty} V^{\operatorname{tri}}(q)=g_{0}^{2} \lim _{w_{2} \rightarrow+\infty} \sum_{j<k} \frac{e^{2 \kappa w_{2}}}{\sinh ^{2}\left(\kappa w_{2}(k-j)+\frac{1}{2} \hat{q}_{j k}\right)}= \\
=g_{0}^{2} \sum_{j=1}^{n-1} e^{-\hat{q}_{j, j+1}}=g_{0}^{2} \sum_{j=1}^{n-1} e^{\hat{q}_{j+1}-\hat{q}_{j}} .
\end{gathered}
$$

This is the potential of the Toda-chain, but the chain is not closed: there is no term $e^{\hat{q}_{1}-\hat{q}_{n}}$ in $V_{0}^{\mathrm{TC}}$.

There is, however, a loophole in the above reasoning: one should better make the substitution (4.37) in the full Calogero potential, $V^{\text {ell }}(q)=-g^{2} \sum_{j<k} \wp\left(q_{j k}\right)$, not in its limit $V^{\text {tri }}(q)$. Then the finiteness of the potential implies that $\left|\kappa w_{2}(k-j)+M w_{2}\right| \geq\left|\kappa w_{2}\right|$ fro all $1 \leq j<k \leq n$ and all $M$. This inequality is usually saturated only for $M=0,|j-k|=1$, but for the single specific value of scaling index $\kappa=\frac{1}{n}$ an extra opportunity arises: $M=-1, k-j=n-1$, i.e. $j=1, k=n$. This provides the lacking term in the potential:

$$
V^{\mathrm{TC}}(\hat{q})=\left.\lim _{\tau \rightarrow i \infty}\left(V^{\mathrm{ell}}(q)+\frac{n(n-1)}{2} g^{2}\left(\frac{\pi}{i w_{1}}\right)^{2} C(\tau)\right)\right|_{\substack{\kappa=\frac{1}{n} \\ w_{1}=-i \pi}}=V_{0}^{\mathrm{TC}}(\hat{q})+g_{0}^{2} e^{\hat{q}_{1}-\hat{q}_{n}} .
$$

Thus the scaling limit exists, provided $g \sim e^{-i \kappa \pi \tau}=e^{-\frac{i \pi \tau}{n}}$. Together with the identification (4.28) this implies that what is finite as $\tau \rightarrow i \infty$ is the product $g_{0}^{2 n} \sim g^{2 n} e^{2 \pi i \tau} \sim m^{2 n} e^{2 \pi i \tau}$ - in nice agreement with (2.1), since $b_{1}=2 \mathrm{~h}_{S L(n)}^{\vee}=2 n$. Note that in order to take the limit in (4.39), we had to add a $q$-independent piece, proportional to $C(\tau)$, to $V^{\text {ell }}$ (or, what is the same, to the Hamiltonian $h_{2}^{N=4}$ ). In other words, the scaling limit involves also redefinition of the moduli: $h_{k}^{N=4}$ are not just the same as $h_{k}^{N=2}$. As soon as the mass $m$ is kept finite, there is a shift: ${ }^{8}$

$$
\begin{aligned}
& h_{2}^{N=2}=h_{2}^{N=4}+\frac{n(n-1)}{2} \frac{m^{2}}{8} C(\tau), \\
& h_{3}^{N=2}=h_{2}^{N=4}+n(n-1) \frac{m^{2}}{8} C(\tau),
\end{aligned}
$$

\footnotetext{
${ }^{8}$ Unfortunately, here we find some disagreement with [3]. In the case of $S L(2)(n=2)$ [3] suggests a formula for $h_{2}^{N=2}-h_{2}^{N=4}$ (it is $\frac{1}{2}(u-\tilde{u})$ in notations of [3], eq.(16.25)) which differs from (4.40) by a substitution of $C(\tau)$ by $\frac{1}{2} \hat{e}_{1}(\tau)=\frac{1}{2}\left(\frac{w_{1}}{\pi}\right)^{2} e_{1}^{0}(\tau)=\frac{1}{6}\left(\theta_{00}^{4}+\theta_{01}^{4}\right)=\frac{1}{3}\left(\theta_{00}^{4}-\frac{1}{2} \theta_{10}^{4}\right)=\frac{1}{3}\left(1-24 \sum_{M \geq 1} \frac{(-)^{M} e^{2 \pi i M \tau}}{\left(1-e^{2 \pi i M \tau}\right)^{2}}\right)=2 C(2 \tau)-C(\tau)$. Though very similar, this is not our $C(\tau)$ in (4.36). The reasoning of [3] at this particular point is somewhat obscure for us: it is not clear, whether within that approach one can determine anything but the value of $\frac{1}{2} e_{1}^{0}(\tau=+i \infty)=$ $C(\tau=+i \infty)$. Note, that in varience with $e_{1}^{0}(\tau)$, our $C(\tau)$ does not have nice modular properties.
} 


\section{Conclusion}

To conclude, we reviewed the present stage of solid knowledge at the "boundary of sciences": between supersymmetric confinement theory and integrability theory. Many interesting, but not yet fully understood speculations, are not included. Moreover, we carefully avoided any discussion of the ways, which led to discovery of Seiberg and Witten, in particular there was no mentioning of $S$-duality. We did the same in the section, devoted to integrability theory. Our goal was to extract the results of both approaches, and make it clear that they indeed coincide. The evidence for this coincidence is striking, while its origin is obscure, and hopefully this should stimulate more people to pay attention to the role of integrability (and other string theory implications) in the modern understanding of non-linear quantum dynamics.

As to straightforward developements on the lines of the present paper, they include examination of Hamiltonian flows, Whitham flows, their relation to (geometrical) quantization, the theory of prepotentials and "quasiclassical $\tau$-functions" and - in somewhat orthogonal direction - generalizations to affine algebras, which from the "target space" perspective should take us from the $4 d$ SUSY field theories to superstrings. At even simpler level, we mention two kinds of issues, not yet identified properly in the context of integrability theory: the $N=2$ SUSY models with matter in the fundamental (perhaps, also some gauge theories with softly broken $N=2 \mathrm{SUSY}$ ), and reinterpretation of the $N=4 \longrightarrow N=2$ flow in terms of Calabi-Yau manifolds [30]. There are many folklor ideas about all these subjects, but we leave uncomplete results beyond the scope of these notes.

\section{Acknowledgements}

We are indebted for discussions to O.Aharony, E.Akhmedov, L.Alvarez-Gaume, S.Das, A.Gerasimov, C.Gomez, A.Gorsky, A.Hanany, I.Krichever, H.Kunitomo, A.Marshakov, A.Mironov, K.Ohta, M.Olshanetsky, A.Rosly, V.Rubtsov, J.Sonnenschein, A.Stoyanovsky and A.Tokura.

H.I. is supported in part by Grant-in-Aid for Scientific Research (07640403) from the Ministry of Education, Science and Culture, Japan. A.M. acknowledges the hospitality of Osaka University and support of the JSPS. 


\section{References}

[1] See, for example,

A.Morozov, String Theory, what is it, Rus.Physics Uspekhi 35 (1992) 671 (v.62, No.8, p.84175 of Russian edition);

A.Morozov, Integrability and Matrix Models, ibid. 164 (1994) No.1, 3-62 (Rus.ed.), hepth/9303139.

[2] N.Seiberg and E.Witten, Electric-Magnetic Duality, Monopole Condensation and Confinement in $N=2$ Supersymmetric Yang-Mills Theory, Nucl.Phys. B426 (1994) 19-52; Err.: ibid. B430 (1994) 485-486, hep-th/9407087.

[3] N.Seiberg and E.Witten, Monopoles, Duality and Chiral Symmetry Breaking in $N=2$ Supersymmetric QCD, ibid. B431 (1994) 484-550, hep-th/9408099.

[4] A.Gorsky, I.Krichever, A.Marshakov, A.Mironov et al., Integrability and Exact Seiberg-Witten Solution, Phys.Lett. 355B (1995) 466-474, hep-th/9505035.

[5] E.Martinec and N.Warner, Integrable Systems and Supersymmetric Gauge Theories, hepth/9509161.

[6] T.Nakatsu and K.Takasaki, Whitham-Toda Hierarchy and $N=2$ Supersymmetric Yang-Mills Theory, hep-th/9509162.

[7] T.Eguchi and S.K.Yang, Prepotentials of $N=2$ Supersymmetric Gauge Theories and Soliton Equations, hep-th/9510183.

[8] R.Donagi and E.Witten, Supersymmetric Yang-Mills Theory and Integrable Systems, IASSNSHEP-95-78, hep-th/9510101.

[9] E.Martinec, Integrable Structures in Supersymmetric Gauge and String Theory, hepth/9510204.

[10] A.Gorsky and A.Marshakov, Towards Effective Topological Gauge Theories on Spectral Curves, ITEP/TH-9/95, hep-th/9510224.

[11] E.Martinec and N.Warner, Integrability in $N=2$ Gauge Theory: A Proof, hep-th/9511052.

[12] A.Klemm, W.Lerche, S.Theisen and S.Yankielowicz, Simple Singularities and $N=2$ Supersymmetric Yang-Mills Theory, Phys.Lett. 344B (1995) 169, hep-th/9411048. 
[13] P.Agyres and A.Faraggi, The Vacuum Structure and Spectrum of $N=2$ Supersymmetric SU $(N)$ Gauge Theory, Phys.Rev.Lett. 73 (1995) 3931, hep-th/9411057.

[14] U.H.Danielsson and B.Sundborg, The Moduli Space and Monodromis of $N=2$ Supersymmetric SO $(2 R+1)$ Yang-Mills Theory, hep-th/9504102.

[15] A.Brandhuber and K.Landsteiner, On the Monodromies of $N=2$ Supersymmetric Yang-Mills Theory with Gauge Group $S O(2 n)$, hep-th/9507008.

[16] A.Hanany and Y.Oz , On the Quantum Moduli Space of Vacua of $N=2$ Supersymmetric $S U\left(N_{c}\right)$ Gauge Theories, hep-th/9505075.

[17] D.Olive and E.Witten, Supersymmetry Algebra that Includes Topological Charges, Phys.Lett. 78B (1978) 97-101.

[18] The classical review paper on integrable systems of particles is: M.Olshanetsky and A.Perelomov, Classical Integrable Finite-Dimensional Systems Related to Lie Algebras, Phys.Rep. 71C (1981) 97-101.

[19] The relevant chapter of integrability theory is very old and the number of references is very large. We mention just a few papers, directly related to our consideration:

I.Krichever, Methods of Algebraic Geometry in the Theory of Nonlinear Equations, Sov.Math.Surveys, 32 (1977) 185-213;

I.Krichever, The Integration of Non-linear Equations by the Methods of Algebraic Geometry, Funk.Anal. and Appl. 11 (1977) No.1 15-31 (Rus.ed.);

I.Krichever, Elliptic Solutions of the Kadomtsev-Petviashvili Equation and Integrable System of Particles, Funk.Anal. and Appl. 14 (1980) 282-290 (No.4 15-31 of Rus.ed.);

B.Dubrovin, Theta Functions and Non-linear Equations, Sov.Math.Surveys, 36 (1981) No.2 11-80 (Rus.ed.);

H.Flaschka and D.McLaughlin, Canonicaly Conjugate Variables for the KdV Equation and the Toda Lattice with Periodic Boundary Conditions, Progr.Theor.Phys. 55 (1976) 438-456;

M.Adler and P.van Moerbeke, Completely Integrable Systems, Euclidean Lie Algebras and Curves, Adv.Math. 38 (1980) 267-317;

M.Adler and P.van Moerbeke, Linearization of Hamiltonian Systems, Jacobi Varieties and Representation Theory, ibid. 318-379.

O.Babelon, E.Billey, I.Krichever and M.Talon, Spin Generalization of the Calogero-Moser System and the Matrix KP Equation, hep-th/9411160. 
[20] The modern-style language in this field uses the notion of Hitchin systems, which empasises interpretation of Lax equation as a flat connection condition. A few directly relevant references are:

N.Hitchin, Stable Bundles and Integrable Systems, Duke Math.Journ. 54 (1987) 91-114;

N.Hitchin, Flat Connections and Geometric Quantization, Comm.Math.Phys. 131 (1990) 347380 ;

E.Markman, Spectral Curves and Integrable Systems, Comp.Math. 93 (1994) 255-290;

A.Beilinson and V.Drinfeld, Quantization of Hitchin's Fibration and Langlands Program, preprint (1994);

B.Feigin and E.Frenkel, Affine Kac-Moody Algebras at the Critical Level and Gelfand-Dikii Algebras, Int.J.Mod.Phys. A7, Suppl.1A (1992) 197-215;

B.Enriquez and V.Roubtsov, Hitchin Systems, Higher Gaudin Operators and R-Matrices, alggeom/9503010;

N.Nekrasov, Holomorphic Bundles and Many-Body Systems, hep-th/9503157;

M.Olshanetsky Generalized Hitchin Systems and the Knizhnik-Zamolodchikov-Bernard Equation on Elliptic Curves, hep-th/9510143;

O.Sheinman, hep-th/9510165;

see also [8] and [9].

[21] See the following papers for general description and references:

B.Dubrovin and S.Novikov, Sov.Math. Surveys 36 (1981) No.2 12 (Rus.ed.);

ref.[23] below.

[22] B.Dubrovin, Geometry of $2 d$ Topological Field Theories, SISSA-89/94/FM;

B.Dubrovin, Integrable Systems in Topological Field Theory, Nucl.Phys. B379 (1992) 627-689;

B.Dubrovin, Hamiltonian Formalism of Witham-type Hierarchies in Topological LandauGinzburg Model, Comm.Math.Phys. 145 (1992) 195.

[23] I.Krichever, The $\tau$-function of the Universal Whitham Hierarchy, Matrix Models and Topological Field Theories, hep-th/9205110;

I.Krichever, The Dispersionless Lax Equations in Topological Minimal Models, Comm. Math.Phys. 143 (1992) 415-429.

[24] A.Losev and I.Polyubin, JETP Lett. 58 (1993) 573, hep-th/9305079.

[25] S.Kharchev, A.Marshakov, A.Mironov et al., Landau-Ginzburg Topological Theories in the Framework of GKM (Generalized Kontsevich Model) and Equivalent Hierarchies, 
Mod.Phys.Lett. A8 (1993) 1047, hep-th/9208046;

S.Kharchev and A.Marshakov, Int.J.Mod.Phys. A10 (1995) 1219.

[26] J.Sonnenschein, S.Theisen and S.Yankielowicz, On the Relation between the Holomorphic Prepotentials and the Quantum Moduli in SUSY Gauge Theories, hep-th/9510129.

[27] A Ceresole, R.D'Auria and S.Ferrara, Phys.Lett. 339B (1995) 71;

A.Klemm, W.Lerche and S.Theisen, Nonperturbative Effective Actions of $N=2$ Supersymmetric Gauge Theories, hep-th/9505150;

[28] See, for example:

M.Abramowitz and I.Stegun, Handbook on Mathematical Functions, Dover Publ., NY, 1965; sect.18.

[29] V.Inosemtsev, The Finite Toda Lattices, Comm.Math.Phys. 121 (1989) 629-638.

[30] See, for example:

A.Strominger, Massless Black Holes and Conifolds in String Theory, hep/th 9504090;

S.Kachru, A.Klemm, W.Lerche, P.Mayr and C.Vafa, Nonperturbative Results on the PointParticle Limit of $N=2$ Heterotic String Compactification, hep/th 9508155;

and ref.[11]. 